\title{
Entretien avec Magda Carneci
}

\section{Propos recueillis le 12 novembre 2014}

\section{Efstratia Oktapoda}

E. O. : Magda Carneci, vous êtes une personnalité connue dans les cercles littéraires en France et en Roumanie, poétesse de renom, artiste sans frontières, écrivaine, traductrice, critique d'art, commissaire d'expositions, et une femme de volonté avant tout. Vous êtes présidente du Club des écrivains de Roumanie (PEN Club Romania), présidente du «Group for Social Dialogue » de Bucarest, professeure associée à la National University of Arts à Bucarest et directrice d'ARTA magazine of visual arts, également à Bucarest. J'ai fait votre connaissance à l'Institut culturel roumain en 2007 , lors d'un cycle poétique que vous organisiez autour des poètes et universitaires français et roumains alors que vous étiez la Directrice du Centre. On a envie de vous connaître un peu plus, connaître votre parcours et ce qui vous a amené en France. Le destin ? S'il fallait vous présenter aujourd'hui au grand public, comment définiriez-vous votre parcours existentiel et professionnel et les raisons qui vous ont amenée en France ?

M. C. : J'ai commencé mon parcours professionnel double, d'écrivaine et d'historienne d'art, dans les années quatre-vingts, quand, en dépit du cloisonnement politique oppressif de la Roumanie sous Ceausescu, une nouvelle génération culturelle prenait toutefois son essor, la génération nommée « quatre-vingts », devenue entretemps très connue là-bas, dont j'ai fait partie en tant que poète et théoricienne. Mon destin semblait devoir se dérouler uniquement en Roumanie. Puis, en raison du fait d'avoir participé activement à la Révolution de décembre 1989 (j'ai été membre du Front du salut national, première structure légitime de pouvoir instituée juste ces jours-là), j'ai été invitée à Paris en février 1990 afin de prendre part à une « Rencontre des jeunes révolutionnaires de l'Europe de l'Est », patronnée par le Président François Mitterrand. C'était le coup de foudre, je suis tombée amoureuse de Paris, en dépit du fait qu'avant, j'étais plutôt orientée vers le monde culturel anglo-saxon. Ensuite, j'ai gagné une bourse du gouvernement français en vue de préparer un doctorat, ce qui m’a permis de venir étudier à Paris pendant deux ans. Ce fut le bonheur, à l'École des hautes études en sciences sociales à Paris. J'ai connu des professeurs tels Jacques Derrida, Cornelius Castoriadis, Louis Marin, Pierre Bourdieu, Alain Besançon, Marc Ferro et autres. J'ai soutenu ma thèse en 1997 à la même école, avec 'magna cum laudae' ; néanmoins, pendant toute la décennie quatre-vingt-dix j'ai vécu à 
Bucarest. Ce fut la période la plus pleine, la plus intense de ma vie, car j'ai publié plusieurs livres de poésie, d'essais et de critique d'art, j'ai beaucoup voyagé à l'étranger grâce à des bourses de recherche, notamment les bourses Fullbright, Getty et Soros, toutes trois aux ÉtatsUnis. J'ai organisé de grandes expositions d'art et, surtout, en tant que membre du Groupe de Dialogue Social (un groupe d'intellectuels roumains engagés), j'ai pu participer aux débats politiques, sociaux et culturels majeurs dans la Roumanie de l'époque.

E. O. : Donc, vous avez quitté la France pour les États-Unis. Mais ensuite, vous êtes revenue en France, puis installée, en tant que docteur en histoire de l'art cette fois. C'était à quelles dates ?

M. C. : C'est à partir de 2001 que je suis revenue en France, j'ai été invitée à donner des cours à l'Institut national de langues et civilisations orientales, à l'INALCO, à Paris. Et voici que mon destin a pris tout d'un coup une tournure française. Pendants ces années vécues en France, j'ai continué à publier des études, des essais et des poèmes, cette fois en français, j'ai organisé un colloque international sur le poète et philosophe Benjamin Fondane, j'ai été le commissaire d'une grande exposition à Salonique sur les arts visuels des Balkans en 2004, j'ai édité un numéro de la revue Poésie 2003 consacré à la poésie roumaine contemporaine et j'ai co-dirigé un ouvrage collectif intitulé Perspectives roumaines. Du post-communisme à l'intégration européenne, paru en 2004. Donc, mon parcours professionnel double, d'écrivaine et d'historienne d'art, s'est continué de manière conséquente dans l'espace français. En 2007, j'ai eu même la joie de voir ma thèse publiée sous le titre Art et pouvoir en Roumanie 1945-1989 chez L'Harmattan.

E. O. : Vous avez plusieurs casquettes, politique et diplomatique, vous n'êtes pas uniquement consacrée au monde universitaire.

M. C. : En effet, après ce parcours universitaire et la publication de ma thèse, tout d'un coup, ma trajectoire a changé de nouveau, car j'ai accepté d'entrer dans la diplomatie, entre 2007 et 2010 j'ai travaillé en tant que directrice de l'Institut culturel roumain de Paris. Ce fut un autre type d'expérience, car j'ai dû endosser pour un temps la posture d'un haut fonctionnaire d'État, responsable de la présentation et de la dissémination de la culture roumaine en France, ce qui m'a obligé à un mode de rigueur institutionnelle et de responsabilité bureaucratique que je ne connaissais pas avant. Parfois ce fut fascinant, parfois ce fut dur, très dur, mais en tout cas je 
pense avoir réussi à accomplir mon rôle assez bien, étant donné qu'à présent il y a des programmes et des résidences de création que j'avais mis en route alors et qui fonctionnent toujours très bien, même si la situation générale des instituts culturels roumains à l'étranger a changé entretemps.

E. O. : Pouvez-vous nous en dire un peu plus de ces programmes et de ces résidences d'artistes, des artistes roumains je suppose?

M. C. : Ce dont je suis assez fière, ce sont les deux résidences de création pour les artistes roumains et surtout le Prix international de littérature francophone Benjamin Fondane que j'ai créé en 2006, qui est accordé désormais chaque année à un écrivain qui écrit en français sans être né citoyen français, selon l'exemple de ce grand penseur et poète français né en Roumanie et mort à Auschwitz en 1944. Je suis aussi fière du congrès international «L'intellectuel en Europe 1989-2009 », que j'ai organisé au Sénat à Paris en novembre 2009, avec d'autres instituts culturels de l'Europe centrale et orientale.

E. O. : Mais vous êtes partie de nouveau, vous avez quitté la France alors que le succès vous suivait partout et que vous deveniez une écrivaine confirmée. Est-ce la nostalgie du pays, ou y at-il d'autres raisons?

M. C. : Je suis partie, oui... La ligne de mon évolution a changé encore une fois. Entre 2010 et 2013 je suis retournée à Bucarest, ma ville natale. Ce fut une décision difficile à prendre mais finalement nécessaire, je dirais. Après quatre ans de travail diplomatique je me sentais vidée de moi-même et en danger de voir sécher mes ressources créatives. Donc, je suis rentrée dans mon pays natal pour me ressourcer, pour retrouver une nouvelle vitalité et pour nourrir en moi certaines dimensions plus intimes, plus fines de mon être. À Bucarest, j'ai publié en 2011 mon premier roman, $F E M$, qui fut un succès et fut nominé pour quatre prix littéraires nationaux. Puis, en 2012, vit le jour le recueil de poèmes TRANS en roumain et, en 2013, le recueil Chaosmos en français aux éditions de Corlevour.

Cependant, début 2014, par une nouvelle boucle de mon destin instable et étrange, je rentre de nouveau à Paris, où j'espère rester cette fois plus longtemps, peut-être pour toujours, sans toutefois rompre mes liens profonds avec la Roumanie. Je ne déchiffre pas encore le sens de ces méandres existentiels, même si je soupçonne vaguement leur but... 
E. O. : Magda, comment vous définissez vous vous-même ? On l'a déjà dit, vous avez plusieurs casquettes. Mais qu'est-ce que vous êtes finalement, diplomate, poète ou intellectuelle, dans tout le sens du terme bien sûr?

M. C. : En tant qu'intellectuelle roumaine vivant entre Paris et Bucarest, je me vois comme «passeur de spiritualité » depuis la Roumanie vers la France et comme «passeur de rationalité » depuis la France vers la Roumanie. En tant que diplomate en charge de la culture, je me définirais comme «médiateur » entre deux espaces nationaux très différents l'un de l'autre et pourtant complémentaires, qui pourraient s'enrichir réciproquement en vue d'une possible synthèse européenne future. En tant que poète, je me perçois plutôt comme une sorte d'«illimiteur de conscience », car c'est comme cela que je conçois le rôle du poète. Mais, s'il fallait donner une seule définition pour mettre ensemble toutes mes occupations et préoccupations, tous mes rêves et obsessions, je me définirais moi-même comme une sorte d' « aventurière spirituelle », une sorte de chercheuse qui a comme but une forme de synthèse entre multiples déterminations et conditionnements apparemment irréconciliables, qui doivent être intégrés créativement - ou bien comme une exploratrice d'une nouvelle manière de vivre vers plus de conscience et de présence au monde et à ce qui le traverse, le transfigure et le transcende.

E. O. : Parlez-nous un peu de votre expérience française. Vous êtes-vous intégrée facilement en France? Avez-vous de difficultés pour vous affirmer, pour vous confirmer, pour vous construire?

M. C. : À Paris, j’ai vécu dès le début parmi des Français, je me suis plongée dans la «soupe humaine locale » avec une certaine frénésie et avec une certaine inconscience. L'adaptation à un autre milieu d'existence n'est jamais facile à digérer, comme on le sait, c'est plutôt traumatique. Il s'agit, en fait, d'une déconstruction et d'une reconstruction de sa vie, à une vitesse accélérée et avec des dangers psychiques inconnus à l'avance. C'est comme une «re-création » de tout ton être, et non pas seulement de la langue dans laquelle on s'exprime. Je comparerais ce processus douloureux à une sorte d'« initiation spirituelle», si on prend une formule ancienne, mais une initiation à une «nouvelle vie » ou une « autre vie » qui se passe dans la solitude, brutalement et sans guide ou maitre, juste avec l'instinct de conservation et la force intérieure de chacun... J'ai 
décrit ce processus plus largement dans un essai qui fut assez prisé à son temps, «Le voyage d'études comme voyage spirituel... » qu'on peut lire aussi sur mon site internet.

En dépit de bonheurs intellectuels et culturels, je me suis sentie à Paris, pendant de longues années, comme dans un exil intérieur assez dramatique, surtout que j'étais poète et que le poète a un commerce très intime avec sa langue natale, qu'il ou elle ne peut pas quitter si facilement. En raison de cet exil intérieur dans une autre culture, j'ai pu réaliser plus vite et avec plus d'acuité l'exil encore plus grand qu'est notre existence, ce fait de «tomber dans le monde » dont parlait Cioran. Il faut le dire, ce fut pour moi une épreuve assez troublante, parfois déchirante, qui, tout en me marquant indélébilement au tréfonds de moi-même, m'a également transformée en un être plus flexible, plus complexe, je dirais plus mature, et surtout plus ouvert à une évolution personnelle accélérée, par rapport à l'être monoculturel que j'étais avant ma fusion avec la France.

Puis, avec le temps et avec le balancement répété entre les deux espaces culturels qui m'ont disputée, la France et la Roumanie, je suis arrivée à dépasser progressivement cette rupture intérieure, ce dépaysement traumatique. À présent, je me sens plutôt bien partout, et à Paris et à Bucarest, et n'importe où dans le monde, comme un citoyen européen ou (de plus en plus) planétaire, pourvu que je garde un contact vivant et constant avec mon être intérieur (qui parle parfois en roumain, parfois en français, parfois, ces derniers temps, en anglais)...

E. O. : Quel était le contact que vous avez eu avec la société française, est-ce que vous trouvez qu'il y a des points communs avec la société et la culture roumaines ?

M. C. : L'expérience française fut pour moi un contact intime avec le mode de fonctionnement d'une société mature, rationnelle et ambitieuse, comme la société française. Par comparaison, la culture roumaine moderne me semble encore jeune, parfois complexée, parfois timorée, et surtout émotionnelle jusqu'au passionnel et au pathétique. En France, on apprend à se contrôler en tant qu'individu social et à se discipliner en tant qu'individu professionnel, car là les standards sont clairs et fermes. En France, on perd l'habitude du vague, de l'impulsivité, de la commodité, comme cela peut arriver en Roumanie, car là on est obligé de fonctionner compétitivement à l'intérieur d'une machine sociale énorme, bien réglée depuis longtemps et très exigeante. En France, j'ai eu l'impression que les individus sont obligés, par les structures qui se sont créées, de fonctionner rationnellement et pragmatiquement, sans illusions et sans complaisances, dans 
une mesure beaucoup plus grande qu'en Roumanie où domine encore une mentalité plus légère, plus superficielle et une affectivité moins censurée dans les relations des gens ou dans les relations professionnelles et publiques.

C'est étrange, mais j'observe que lorsque je vis à Paris je pense de manière plus logique, plus intentionnelle et plus constructive. Dès que je rentre à Bucarest, j'observe que je reviens à une pensée principalement émotionnelle, voire passionnelle, plutôt vague et inclinée vers le négatif, le dramatique, typique pour les Roumains. Entre les deux formatages mentaux, je sens parfois une tension, une incompatibilité, un refus réciproque qui parfois me disperse. Mais j'observe aussi comment, avec le temps, cette tension tend à diminuer, à s'effacer... J'espère pouvoir réaliser dans l'avenir prochain une synthèse entre les deux modes de capter la réalité et d'envisager l'existence, que représentent les deux cultures que j'adore et qui me disputent.

E. O. : Vous êtes entre deux cultures, vous vivez aussi entre deux cultures, laquelle vous préférez, quelle est la culture qui vous exprime, qui vous inspire?

M. C. : Me situant à mi chemin entre l'une et l'autre, et les regardant d'une manière plutôt détachée à présent, je ne peux pas m'empêcher d'observer les avantages et les désavantages des deux côtés. Je pense que la culture du rationnel devrait être équilibrée par la culture de l'émotionnel, et vice-versa. Il serait merveilleux si on savait appliquer de manière différenciée ces deux modalités d'exister dans le monde - si, par exemple, les Roumains utilisaient la « vision française » dans les relations socioprofessionnelles et si les Français utilisaient la «vision roumaine » dans les relations interhumaines et dans le domaine spirituel. Je sais que ces affirmations sont un peu générales et qu'on pourrait toujours trouver des contre-exemples, mais je sais aussi que mes observations contiennent un grain (ou plusieurs) de vérité. Cependant, avec le temps et le brassage des nationalités dans le cadre de l'Union Européenne, j'ai l'impression que ce mélange et cette synthèse dont je parle, se trouvent déjà en route et nous attend dans l'avenir pas très lointain...

E. O. : Est-ce que la France vous a finalement gagnée, puisque vous avez opté de revenir vivre en France ? Et est-ce que cela ne traduirait pas un détachement psychique, une désaffection de la Roumanie de votre cœur et de vos origines? 
M. C. : Je ne sais pas si mon option pour la France sera un choix pour toujours. Je constate que mon métabolisme psychique et spirituel a besoin de changer de place périodiquement, tous les quatre ans ou presque, pour garder un certain dynamisme et une présence lucide au monde. Je pratiquerai probablement la navette entre les deux cultures, comme le pratiquent à présent pas mal d'artistes et intellectuels internationaux qui ont parfois deux, même trois domiciles différents. Ce n'est pas toujours facile, il faut le reconnaître. Mais nous nous trouvons probablement à l'époque des «aventuriers culturels »- ceux qui essayent de dépasser les limitations géoculturelles traditionnelles, mus non pas par des motivations politiques et économiques ou par une forme quelconque de discrimination, mais plutôt par le besoin de se libérer des conditionnements culturels hérités, inculqués par éducation, pour se dépasser humainement afin de trouver une nouvelle créativité, un nouvel horizon des possibilités humaines... Ces «aventuriers culturels » représentent peut-être les «pontifes cachés » de notre temps, ceux qui créent des liens, des réseaux et des ponts pour l'émergence d'une culture planétaire, pour une nouvelle conception transgressive et donc transnationale de l'humain.

E. O. : Parlons de votre œuvre littéraire et de votre écriture en français. Poète confirmée, vous aimez écrire des poèmes et vous avez une verve philosophique et existentielle.

M. C. : J'ai publié en 1997 un premier recueil de poèmes traduits en français, Psaume, aux éditions Autres Temps à Marseille. À ma surprise, il fut plutôt bien reçu, étant donné que c'était une sélection de poèmes traduits par différents traducteurs et parus dans une maison d'édition non-parisienne. Avec ce petit recueil j'ai réussi à me créer des amitiés parmi quelques poètes français connus et j'ai réussi aussi à ouvrir les portes de quelques revues littéraires et institutions culturelles parisiennes. Grâce à ce recueil, on m'a proposé par exemple de coordonner un numéro de la revue Poésie 2003, numéro qui fut en fait une anthologie de la poésie roumaine actuelle. Le recueil Psaume fut publié, en fait, en collaboration avec les éditions Les Écrits des Forges de Québec, la plus grande maison de poésie francophone du Canada. Ces éditions vendent mon recueil même à présent dans les festivals et les marchés de poésie de l'espace francophone.

Après avoir publié des poèmes dans des revues diverses - telles Action Poétique, Aujourd'hui Poème, Autre SUD etc. - j'ai réussi à publier un deuxième recueil de poèmes, Trois saisons poétiques, en 2008, aux éditions PHI au Luxembourg, dans la collection «Graphiti » 
dirigée par le poète Jean Portante. C'est un recueil qui contient trois cycles : Musique pour instruments inconnus, Voyage spatial et On dirait des psaumes, traduits du roumain en français par Linda Maria Baros, jeune poète francophone d'origine roumaine très talentueuse. Enfin, début 2013, j'ai publié un troisième recueil de poèmes, Chaosmos, aux éditions de Corlevour, toujours dans la traduction de Linda Maria Baros.

E. O. : Est-ce que vous êtes aussi connue aux États-Unis ? Vous avez séjourné aux États-Unis. Avez-vous publié des recueils ou des traductions aux États-Unis ?

M. C. : Les États-Unis me semblent une expérience nécessaire, voire fascinante, car c'est la nouvelle Babylon de notre temps, pour dire vite. Là, je ne suis pas si connue. Même si j'y ai publié un recueil de poèmes, Chaosmos, en 2006, ainsi que de nombreux groupages de poèmes dans plusieurs revues littéraires grâce à mon traducteur fidèle, Adam J. Sorkin, professeur de littérature à l'Université de Pennsylvanie. Les États-Unis sont un grand continent, en effet, et la littérature étrangère représente un pourcentage mineur de ce qui est publié chaque année. De plus, la poésie n'est pas un domaine très juteux, sauf pour les revues littéraires qui pullulent autour des presses universitaires qui, elles, par contre sont nombreuses. D'autre part, je n'ai pas eu le temps ces dernières années de m'occuper de ma poésie outre-mer, son sort européen m'intéresse davantage. Dans ce sens, j'ai publié des poèmes dans plusieurs revues littéraires anglaises et, récemment, j'ai reçu une invitation pour un nouveau recueil de poèmes de la part d'une maison d'édition australienne, ce qui me semble un «challenge » assez attirant et j'ai envie de lui donner cours. Car c'est peut-être un signe que la poésie fonctionne en réseau international, comme tout à présent, et qu'il faut aller là où cette fleur rare trouve un terrain fécond.

E. O. : Magda Carneci, présentez-nous l'ensemble de votre œuvre (poésie, traductions, essais), qu'est-ce que vous aimeriez dire au grand public ?

M. C. : En fait, je travaille et je produits des écrits dans plusieurs domaines, comme vous l'avez compris. Dès mon début, j'ai avancé sur deux voies professionnelles en même temps, j'ai été historienne et critique d'art, d'une part, et, d'autre part, j'ai publié des livres de poésie, des essais, des traductions et de romans. Tout cela, à côté d'un journalisme culturel aux implications politiques et sociales que j'ai pratiqué surtout dans les années quatre-vingt-dix et au début des 
années 2000. Je dirais que j'ai construit une œuvre assez fournie, assez diverse, une œuvre qui s'est proposée, je le constate à présent, à tenter un dépassement du cloisonnement professionnel dans des cases fixes et rigides et la transmission d'une énergie unique pour le dépassement de nos limites intérieures diverses.

E. O. : Madame Carneci, vous parlez un français excellent, vous écrivez en français, des livres, des essais, vous avez aussi écrit des poèmes directement en français. On dirait que c'est inné chez vous. Est-ce que la langue française est aussi importante pour vous ? qu'est-ce qu'elle incarne pour vous? Qu'est-ce que cela fait d'écrire dans une langue « autre »?

M. C. : En tant qu'écrivain, j'ai été tentée modérément de changer ma «patrie linguistique ». Cela est venu parfois de soi et non pas programmatiquement, et cela m'arrive, surgit de moi, de plus en plus fréquemment ces derniers temps. Puisque ma poésie mise moins sur les subtilités de langage et plus sur la vision poétique, soutenue par le rythme, le souffle et par des images qui passent facilement d'une langue à l'autre, cette poésie se traduit assez bien et ne pose pas de grands problèmes aux traducteurs avec lesquels je travaille.

Cependant, je constate que j'écris sans inhibition des textes théoriques en français (et même en anglais), mais pour la poésie j'ai longtemps fait appel aux traducteurs, par une sorte de respect profond pour les résonances subtiles de la langue française. Néanmoins, il m'est arrivé de temps en temps d'écrire des cycles de poèmes directement en français parce que les vers sont descendus en moi justement dans ce revêtement sonore, pendant certains moments rares d'exaltation, ce qui m'a étonné au début et qui m'a remplie de joie par la suite. Depuis peu, je me risque aussi d'écrire des proses directement en français, et c'est une expérience assez intéressante, assez éprouvante.

En tout cas, écrire dans une autre langue reste une expérience troublante et transformatrice, c'est comme si on naissait mentalement de nouveau - et même par rapport à ta langue maternelle, que tu perds pour la retrouver autrement, plus lucidement et plus consciemment. C'est une épreuve enrichissante, finalement. De ce point de vue, l'expérience actuelle de brassage civilisationnel et culturel généralisé au niveau mondial nous met aussi devant un nouveau rapport à la langue, ou aux langues qu'on parle. Je pense qu'il faudrait nous rendre compte que non seulement les frontières extérieures, géographiques ou étatiques tombent ou se subtilisent, comme on dit, avec la mondialisation, mais aussi les frontières intérieures, 
mentales, voire linguistiques, et que nous assistons à présent à un élargissement de nos possibilités humaines, cantonnées très longtemps dans un espace géographique et cérébral réduit. Dans ce sens, assumer personnellement et provoquer chez les autres une «i-limitation» perceptive et intellective devient une sorte de devoir. Les poètes, les artistes en général, sont ou devraient devenir des « illimiteurs de conscience ».

E. O. : La poésie est pour vous une véritable voie, une sorte de figure mythologique qui vous guide pour vous exprimer, pour vous libérer. Que représente la poésie pour vous ?

M. C. : Il m'a fallu un certain temps pour comprendre que, pour moi, la poésie, plus qu'une vocation, une profession, une mission ou une croyance - est tout d'abord une Voie. Qu'elle mène quelque part, ou qu'elle devrait mener quelque part. C'est seulement progressivement, et souvent rétrospectivement, que j'ai remarqué et compris que la manière dont on fréquente les mots peut produire en vous une modification discrète, un changement de plus en plus profond, et finalement une transformation assez inattendue, assez radicale.

C'est comme si, lorsqu'on prend les mots au sérieux et qu'on les vit chacun pour luimême, avec l'océan de profondeur existentielle qui leur correspond, et surtout lorsqu'on se les applique à soi-même, en toute naïveté, en toute sincérité, comme si tous les grands vers du monde vous concernaient personnellement et étaient écrits spécialement pour vous, quelque chose finit par arriver. Quelque chose d'assez miraculeux. Car la vibration (ontologique, émotionnelle ou spirituelle) encapsulée dans les mots ouvre lentement, petit à petit, certains canaux inconnus et mystérieux en vous, avec lesquels elle entre en résonance et qu'elle réveille d'un long sommeil, après une longue attente, en les activant de façon spectaculaire.

Et brusquement, on commence à avoir accès à des sensations plus amples et plus intenses qu'à l'ordinaire. Les impressions du monde sont plus colorées, plus saturées, plus fortes, et toutes sortes de correspondances inattendues s'établissent entre elles dans votre tête. On saisit d'incroyables coïncidences entre la pensée et la réalité extérieure, des synergies insoupçonnées commencent à se mettre en mouvement autour de vous entre les différents plans de l'existence, et, de temps à autre, on a accès à de fulgurants états délimités, à des sentiments universels, lorsqu'on s'identifie à la planète entière et que celle-ci est vivante, vivante, et elle vous transmet en silence son amour enveloppant, protecteur. 
Puis, petit à petit, les «choses du monde», les ambitions, les vanités, les priorités commencent à se hiérarchiser différemment. On commence à comprendre que l'important n'est pas ce qui nous accapare quotidiennement, ce qui nous hypnotise d'habitude, ce qui dévore le peu de temps que nous avons à notre disposition en cette vie. L'important est de percevoir le monde autrement, de se rendre compte qu'il est un étrange assemblage, un décor géant en carton-pâte, une sorte de plateau de tournage d'un jeu cosmique époustouflant, où notre rôle pourrait ne pas être celui que nous nous attribuons, que nous imaginons. Notre rôle pourrait être - et il est peut-être au fond - celui de témoins de cette danse apocalyptique-aurorale, de cet extraordinaire spectacle dramatique-tragique-comique-enthousiasmant-enivrant dans lequel nous sommes acteurs mais aussi spectateurs, cause et effet, appât et enjeu, nous ne sommes rien et nous sommes tout, nous sommes de pauvres cobayes mais aussi des dieux en puissance.

La fréquentation poétique des mots, ou la fréquentation des mots poétiques, ouvre une voie, est une mise en voie, peut-être plus que la Voie elle-même. Celle-ci commence peut-être dans son prolongement, est très étendue et mène beaucoup plus loin. Mais la poésie est une mise en voie à notre portée, abordable, douce, appropriée peut-être pour l'homme contemporain, accablé par la civilisation, extirpé de transcendance. La poésie est une sorte de technique transcendantale, bafouilleuse, déformée, corrompue, à la mesure de l'époque désenchantée que nous vivons, où la flamme du miraculeux est gardée vivante (même si ce n'est qu'à tout petit feu) justement par la poésie. Il s'agit d'une nourriture subtile sans laquelle notre être intérieur mourrait. Car l'être humain, tel que nous le vivons à présent, n'est pas encore accompli, achevé. L'homme véritable, réel, est encore en voie de germination dans l'homme actuel : un homo faber obstiné, devenu de temps en temps homo poieticus, dans son processus méandrique et lent de transformation en homo pertinents, peut-être. Et pour cet homo sui trancendentalis qui attend en nous, la poésie est une substance nécessaire, un combustible à notre portée, même s'il n'est pas suffisant. C'est juste une mise en voie, éventuellement.

E. O. : Votre poésie est une poésie philosophique et profondément existentielle. Est-ce que vous l'avez choisie exprès pour vous exprimer, pour dire ce que vous ne pouviez pas dire autrement ?

M. C. : Je n'aurais pas voulu emprunter cette voie, je n'y étais d'ailleurs peut-être pas destinée. Mais la poésie a été plus forte que moi, car elle m'a entraînée en quelque sorte dans sa voie par l'accès qu'elle m'a donné de bonne heure à une autre perception et compréhension du parcours 
de l'existence dans le monde. C'est pourquoi j'ai vécu la poésie comme une lutte, comme un combat, comme un parcours semé d'obstacles, avec des nœuds gordiens à couper, avec des questions du Sphinx à résoudre, avec des «travaux d'Hercule » à dépasser. Mes recueils ne sont pas nombreux et ne se ressemblent pas beaucoup, même s'ils sont probablement structurés en profondeur par le même souffle et les mêmes obsessions. Mais je n'ai pas pu produire plus, et je n'ai pas pu me répéter, car chaque volume a été le résultat serré, la scorie d'une combustion assez acerbe, la trace scripturale succincte d'une étape close, d'une blessure dépassée, d'une «lutte avec l'ange » laissée ainsi derrière soi.

Je pense qu'il me reste peu d'étapes à parcourir encore sur ce chemin poétique accidenté, peut-être une ou deux encore. Après quoi un autre chemin devra peut-être suivre. Une autre voie, si je veux vraiment que mon existence signifie évolution vers plus-que-moi-même, progression dans l'inconnu, dans le miraculeux, et non enfermement dans une formule existentielle ou professionnelle confortable, protectrice, mais inévitablement bornée par la mort.

Car la poésie véritable devrait ouvrir vers plus-que-la-poésie, si elle doit garder sa fonction originaire de mise en résonance du monde infini en nous avec le monde infini en dehors et dans l'univers ; sa nature ontologique d'ouvreuse de chemins vers plus-que-la-réalité au sein même de la réalité.

E. O. : Quels sont les thèmes majeurs de votre poésie?

M. C. : Je pratique une poésie alluvionnaire, visionnaire, qui se propose d'explorer deux pôles extrêmes du discours poétique : d'une part, l'élément « chaotique », désordonné, fragmentaire et prosaïque de la réalité perçue, et, d'autre part, la dimension «cosmotique » de l'ordre, de la géométrie et de l'intégration, à travers l'esprit, de l'être et du monde dans une nouvelle cohérence. Comme les titres de mes recueils l'indiquent, cette poésie se propose de se situer au point d'orgue d'un équilibre instable entre ces deux états opposés, en les harmonisant via un torrent verbal qui met en jeu un lyrisme post-romantique. La sensation de force visionnaire qui transporte vient du mariage explosif du concret brutal et de l'abstrait immatériel, de la mise en commun du sensuel cru et du spirituel aérien, du terrestre et du cosmique, à travers les techniques du paradoxe et de l'oxymoron, afin d'arriver à une transfiguration de la réalité vécue vers un « chaosmos » intégratif et étonnant. 
E. O. : Quelles sont les thèmes qui vous inspirent et qui caractérisent non seulement votre poésie mais l'ensemble de votre œuvre ?

M. C. : Comme je l'ai déjà dit plusieurs fois, je suis mue par quelques obsessions qui reviennent souvent dans mes écrits et mes prises de position. Je crois profondément que nous nous trouvons, en tant qu'espèce, à un tournant décisif pour notre évolution ou notre involution, voire notre disparition. La quantité énorme de connaissances disponibles, la mise en connexion de toutes les cultures du monde entier, les pressions accrues que subissent les individus et les sociétés, la capacité d'influencer ou de détruire complètement le milieu environnant et beaucoup d'autres signes montrent qu'on approche à un moment où, après l'accumulation quantitative actuelle, un saut qualitatif sera nécessaire, voire une mutation intérieure, de nature psychologique.

Nous vivons un instant critique, mais aussi d'ouverture, où plusieurs révolutions sont en cours, une révolution scientifique et technique, mais aussi une révolution sociale, culturelle, spirituelle; en un mot, un renversement de la civilisation, si on peut dire. Le brassage des ethnies, des cultures et des peuples a probablement porté son coup final, a constitué et constitue toujours un séisme nécessaire pour le dépassement des grandes barrières extérieures et intérieures et pour la transgression de nombreuses contraintes mentales artificielles, imposées par l'histoire et héritées d'elle. Nous nous trouvons au terme d'une époque dominée par la puissante légitimité rationaliste, avec sa logique linéaire et pragmatique de l'univers mental pur et dur, refusant obstinément d'autres modalités complémentaires pour aborder les questions de la nature, de l'homme et du cosmique. La pensée strictement rationnelle qui utilisait des opérations binaires dont la charge holistique et prévisionnelle était drastiquement limitée par ses propres suppositions initiales, commence à faire place, lentement, mais irrépressiblement, à une pensée nourrie également par d'autres dimensions de l'humain, de la dimension psychique d'abord et de celle du supra-mental ensuite.

Il me semble assez évident que nous nous trouvons devant une nouvelle expansion de notre conscience actuelle, grâce aux expérimentations et aux efforts fournis dans de multiples directions : des sciences du micro et du macrocosme à la redécouverte des grandes traditions spirituelles orientales et à leur mise en connexion avec la tradition occidentale, du bombardement informationnel et de la communication instantanée en masse, à la biologie génétique et à la nanotechnologie, du besoin de vivre des expériences «fortes », comme les drogues, le sexe, la musique, le sport de haute performance, à la religiosité, à l'ésotérisme, au développement de soi 
et à la «mindfulness », etc. Toutes ces choses indiquent assez clairement le fait que nous nous trouvons à un moment où nous devons forcer les barrières de la perception et de l'intellect, agrandir le concept de l'humain, le transformer. Mais si nous ne conscientisons pas le sens de cette expérience géante, nous risquons de détruire l'humain. Il s'agit d'une expérience qui se déroule à l'échelle planétaire, dont les poètes et les artistes devraient témoigner avec ténacité et courage, à travers leurs vies, leurs œuvres et leurs prises de position, s'ils veulent garder leur rôle spécifique d' «illuminateurs de conscience » à l'intérieur du métabolisme général de l'humain, et respecter ainsi la « responsabilité visionnaire » dans laquelle ils sont investis.

E. O. : Tout cela est profondément philosophique. Si vous étiez une déesse de la mythologie Madame Carneci, quelle déesse aimeriez-vous être?

M. C. : Il y a longtemps, un critique littéraire m'avais mise, selon la poésie que j'écrivais, sous le signe de la déesse Diane ou Artemis, c'est-à-dire sous le signe d'une féminité fragile et terrible en même temps, cachée et simultanément exhibée, capable d'exploits irrationnels et paradoxaux. Il y avait un grain de vérité dans cette description, mais à présent j'aspire vers la raison lumineuse et claire de la déesse Athéna. Un autre couple de déesses que j’adore est Isis et MarieMadeleine (bien sûr que je sais que la dernière fut un personage réel, transfiguré en symbole) : je me retrouve dans ces deux figures féminines parce que leurs histoires concrétisent de manière différente un même schéma initiatique concernant l'évolution possible du psychisme vers l'harmonisation du féminin et du masculin dans la nature humaine complète et la réalisation du Soi, de sa propre divinisation.

S'il s'agissait de prendre des exemples ou des modèles de l'histoire réelle, je dirais qu'à l'heure présente de mon être toujours en dévenir, je rêve d'un mélange impossible, d'une synthèse improbable entre la reine Elisabeth première d'Angleterre et la sainte Thérèse d'Avilla. C'est-à-dire, reconcilier dans un seul psychisme le pouvoir extérieur avec la force intérieure, la capacité de légiférer dans le monde avec la disponibilité de porter son esprit vers les zones les plus hautes et pures. Autrement dit, marier l'intelligence mondaine, raisonable et ferme, avec l'émotionnalité raffinée jusqu'à l'état mystique. Mais la synthèse de la régalité et de la sainteté, des droits essentiels de l'être humain, est très rarement ou jamais réalisée. 
E. O. : Madame Carneci, vous êtes historienne d'art, avec une thèse de doctorat en France. Quel type d'art vous attire, quel art vous incarne et pourquoi ?

M. C. : En tant qu'historienne d'art, dans un premier temps je me suis spécialisée dans l'art moderne de l'Europe centrale et orientale. Cela est évident dans le thème de ma thèse de doctorat, «Art et pouvoir en Roumanie 1945-1989 », où j’ai étudié les arts visuels roumains pendant le régime communiste et où j'ai mis la situation roumaine en parallèle avec la situation des autres pays communistes de l'Europe de l'Est, comme on disait à l'époque. Tout en étant historienne d'art de formation, je me suis toujours investie, dès le début de ma carrière, dans l'art contemporain de la même zone centrale-est-européenne. J'ai publié plusieurs monographies d'artistes roumains de la première moitié du vingtième siècle, mais j'ai aussi publié des études et des essais sur ce qui s'est passé visuellement dans la même région pendant les trois dernières décennies. Mes livres et mes études ont fourni des informations utiles pour des grandes expositions dédiées à ce sujet telles After the Wall 1999-2000, In the Gorges of the Balkans 2003, ou Les promesses du passé, exposition qui s'est tenue au Centre Pompidou en 2010.

E. O. : En effet, vous êtes une artiste innée. Est-ce que vous faites des peintures ou des sculptures?

M. C. : C'est vrai que j'ai eu deux vocations dès mon adolescence, celle de poète mais aussi celle de plasticienne. Je peignais très bien, j'avais même inventé une technique à moi qui donnait des résultats assez surprenants. Cependant, puisque j'avais aussi l'intérêt pour la poésie et la lecture, j'adorais les livres d'art et d'esthétique, j'ai voulu réconcilier les deux «attirances » en choisissant d'étudier l'histoire de l'art à Bucarest. Ce ne fut pas un choix facile, j'ai continué à peindre pendant plusieurs années dans ma jeunesse estudiantine, puis j’ai abandonné. Je me suis investie par la suite dans la critique d'art et l'interprétation des œuvres des divers plasticiens, roumains et étrangers, pendant de longues années. Par ailleurs, j'ai illustré mon recueil français de 2008, Trois saisons poétiques, avec mes propres photos, et récemment, en septembre 2013, j'ai exposé au Victoria Art Center, une galerie d'art importante de Bucarest, une série de photos de moi-même prise devant des œuvres célèbres du patrimoine pictural roumain. Cette série eut un beau retentissement dans le public. 
E. O. : Et comme artiste, comment vous définissez-vous ?

M. C. : Je préfère le mot « créatrice » à la place de l'appellation « artiste », car c'est plus large, c'est polyvalent et permet plusieurs types d'activités artistiques, tout en restant un peu vague, un peu flou comme notion. En tant que porteuse d'une énergie créatrice de type artistique, je me reconnais dans une citation de Nietzsche qui dit qu' «il y a une similitude naturelle entre le conquérant, le législateur et le poète, car tous les trois partageant une même impulsion de transférer leur énergie créatrice dans la matière ». Quand on est traversé, voire «possédé » par cette énergie, les déterminations nationales ne comptent plus tellement. On se sent « créateur du monde $»$, comme on se sent « citoyen du monde $» . .$.

E. O. : Merci Magda pour ces propos pleins d'humanisme, pour vos créations et votre engagement et les réflexions que vous avez voulu partager avec nous.

\section{Bibliographie de l'auteure}

\section{Volumes de poésie en roumain}

- TRANS. Bucarest : Tracus Arte, 2012.

- Chaosmos et autres poèmes. Bucarest : Paralela 45, 2004.

- Poèmes politiques. Bucarest : Axa, 2000.

- Chaosmos. Bucarest : Cartea Romanesca, 1994.

- Un silence assourdissant. Bucarest : Eminescu, 1984.

- Hypermatière. Bucarest : Cartea Romaneasca, 1980.

\section{Roman}

- FEM. Bucarest : Cartea Romaneasca, 2011.

\section{Recueils de poèmes en français}

- Chaosmos. Np : Corlevour, 2013.

- Trois saisons poétiques. Np : PHI, 2008.

- Psaume. Montréal/Marseille : Écrits des forges/Autres Temps, 1997.

\section{Proses dans des ouvrages collectifs en français}

- Mon royaume pour un livre. 17 écrivains racontent. $\mathrm{Np}$ : Castor astrale/L'atelier imaginaire, 2013.

- La mort aujourd'hui. Paris : Rocher, 2005. 
- Le sacré aujourd'hui. Paris : Rocher, 2003.

- Paris par écrits. Paris : Inventaire, 2002.

\section{Volumes de poésie dans d'autres langues}

- Chaosmos (anglais). New York : White Pine P, 2006.

-Chaosmos (hollandais). Amsterdam : Go-Bos P, 2004.

\section{Traductions}

- Pierre Oster. Alchimie de la lenteur (du français en roumain). Bucarest : Paralela 45, 2007.

- Nicole Brossard. Installations (du français en roumain). Bucarest/Montréal : Paralela 45/Écrits des Forges, 2005.

- Catherine Durandin. Bucarest. Souvenirs et promenades (du français en roumain). Bucarest :

Paralela 45, 2004.

- Christopher Merrill. Only the Nails Remain. Scenes from the Balkanwars 1992-1997 (de l'anglais en roumain). Bucarest : Paralela 45, 2002.

- des poètes français traduits pour les revues roumaines comme : Michel Deguy, Jacques Darras, André Velter, Claude Esteban, Jean-Michel Maulpoix, Henri Deluy, Marie-Claire Bancquart, Jean-Pierre Siméon, Pierre Oster, Alain Lance, Paul Louis Rossi, Marie Etienne, Ariane Dreyfus, Dominique Fourcade, Anne-Marie Albiach, et autres.

- des auteurs roumains traduits en français : Paul Celan, Urmuz.

\section{Livres d'artistes}

- Peau-ésies. Paris : Trans Signum, 2008.

- Le paradis poétique. Paris : Tran Signum, 2004.

\section{Anthologie de poésie en français}

Poésies de langue française. 144 poètes d'aujourd'hui autour du monde. Paris : Seghers, 2008.

\section{Essais}

- Romanian Writers on Writing. Dir. Norman Manea. San Antonio, Texas : Trinity UP, 2011.

- Art et pouvoir en Roumanie 1945-1989, Paris, L'Harmattan, 2007.

- Transdisciplinarité - Un chemin vers la paix, Paris, Éditions FBV pour le CNRS, 2005.

- Perspectives roumaines. Du postcommunisme à l'intégration européenne. Dir. Catherine Durandin et Magda Carneci. Paris : Harmattan, 2004. 


\section{Édition}

- Éditeur-en-chef de la revue d'arts visuels ARTA de Bucarest.

- Directrice de la collection de poésie internationale «Gemini» aux éditions Paralela 45 à Bucarest.

\section{Responsabilités}

- Présidente du Groupe pour le Dialogue Social, Bucarest (depuis 2012)

- Présidente du PEN Club roumain (depuis 2011)

- Membre du Parlement Culturel Européen (depuis 2005)

\section{Prix}

2013 : Prix Opera Omnia, Union des écrivains de Roumanie, section de poésie de la ville de Bucarest.

2000 : Ordre National du mérite culturel, en grade de chevalier.

1997 : Prix pour critique d'art de l'Union des artistes plasticiens de Roumanie.

1993 : Prix pour critique d'art de l'Association des collectionneurs d'art de Roumanie.

1992 : Prix de poésie de la ville de Slobozia.

1986 : Prix de poésie de la revue culturelle Ateneu.

\section{Sites internet :}

www.magdacarneci.ro

www.m-e-l.fr

www.kulturparlament.com

www.penromania.ro

www.gds.ong.ro 